\title{
Biomimetic piezoelectric quartz sensor for folic acid based on a molecular imprinting technology
}

\section{Rashmi Madhuri, Mahavir Prasad Tiwari, Deepak Kumar, Aparna Mukharji, Bhim Bali Prasad}

\author{
Department of Chemistry, Faculty of Science, Banaras Hindu University, \\ Varanasi 221 005, India
}

*Corresponding author. Tel: (+91) 945-1954449; Fax: (+91) 542-22368127, E-mail: prof.bbpd@yahoo.com

Received: 25 Dec 2010, Revised: 01 March 2011 and Accepted: 05 March 2011

\section{ABSTRACT}

A novel molecularly imprinted polymer (MIP)-modified quartz crystal microbalance (QCM) sensor with high selectivity has been developed for the determination of folic acid via activator generated-atom transfer radical polymerization (AGET-ATRP) technique. It requires an alkyl halide $(\mathrm{R}-\mathrm{X})$ as an initiator, a transition metal complex as a catalyst, and an amine as reducing agent. Herein, chlorosilane was used as initiator which was grafted onto the self assembled monolayer modified-quartz crystal surface followed by the addition of pre-polymer mixture which latter underwent thermal cross-linking resulting in MIP-modified QCM sensor. The linear working range (quantification) was found to be $0.6-26.0 \mu \mathrm{g} \mathrm{L}^{-1}$, with the detection limit as low as 0.08 $\mu \mathrm{g} \mathrm{L} \mathrm{L}^{-1}(\mathrm{~S} / \mathrm{N}=3)$. Copyright (C) 2011 VBRI press.

Keywords: Molecularly imprinted polymer; quartz crystal microbalance; folic acid; activator generated-atom transfer radical polymerization.
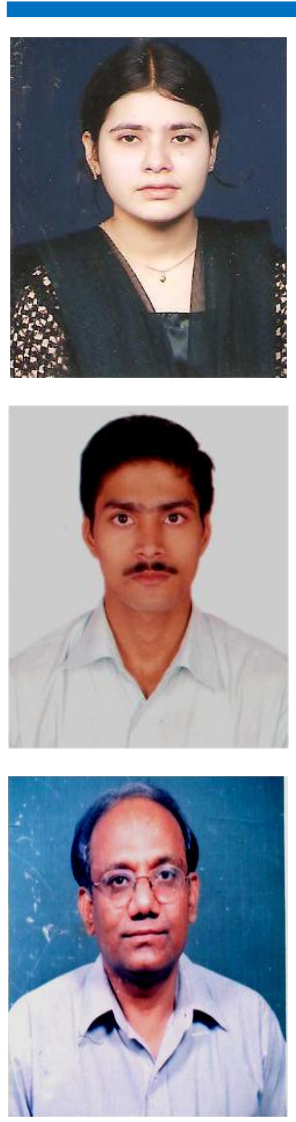

Rashmi Madhuri is currently pursuing a $\mathrm{Ph} . \mathrm{D}$. at Banaras Hindu University under the supervision of Dr. Bhim Bali Prasad. She received her B.Sc. in 2005 and M.Sc. in 2007 from Banaras Hindu University, India. She is recipient of CSIR NET Junior Research Fellowship and BHU research fellowship. Her research interests lie in the field of chemical sensor development, molecularly imprinted biomimetic polymers, and electroanalytical chemistry.

Mahavir Prasad Tiwari is a research scholar in Department of Chemistry, faculty of Science, BHU, India under the supervision of Dr. Bhim Bali Prasad. He received his B.Sc. in 2005 and M.Sc. in 2007 from Purvanchal University. His research interests lie in the field of solid phase extraction/microextraction, molecularly imprinted polymers, and electroanalytical chemistry.

Bhim Bali Prasad is currently a professor at the Banaras Hindu University, India where he has mentored $20 \mathrm{Ph} . D$. students and published 90 research papers in several reputed international and national journals. $\mathrm{He}$ received his B.Sc. degree in chemistry in 1972 and M.Sc. degree in 1974 form Banaras Hindu University, India. He obtained his $\mathrm{Ph} . \mathrm{D}$. from the Department of Chemistry, faculty of Science, BHU, India with Dr. Lal Mohan Mukherjee. He is a recipient of several national awards for his research contribution in analytical chemistry. $\mathrm{He}$ also went to Ranbaxy Ltd., India for about one-and-half year and elaborated a protocol for pharmaceutical analysis, interfaced with several sophisticated instruments. His research interests include environmental chemistry, chromatography, electroanalysis, and detection principle for chemical analysis and development of biomimetic chemical sensor using molecularly imprinted polymers for clinical, pharmaceutical and biological analyses.

\section{Introduction}

Quartz crystal microbalance (QCM) is a simple, low-cost, high-resolution mass sensing technique [1], however, there is no specific selectivity. As a result, various chemicals and biomaterials have been used to modify (physically or chemically) the QCM surface in an effort to obtain selectivity. In recent decades, pre-coated (QCM)-based piezoelectric immuno-sensors have received widespread applications in the analysis of clinical targets [2], the monitoring of environmental contaminants, such as pathogen and bacteria [3] and the detection of biomolecular interaction [4]. This ensured attractive performance, such as high specificity, low cost, ease of use, and rapidness of detection. The most attractive one is molecular imprinted polymers (MIPs) to obtain a selective polymer layer on the surface of QCM [5]. Molecular imprinting is a method for making selective binding sites in synthetic polymers using molecular template. Target molecules can be used as template for imprinting the cross-linked polymer. After the 
removal of the template, the remaining polymer is selective for template. However, to the best of our knowledge, only a few applications of MIPs in QCM sensor have been reported and moreover most of them are only confined to biomolecules recognition [6]. However, there is hitherto none MIP-QCM reported for folic acid (FA) sensing. FA is a water-soluble vitamin and its deficiency can cause several chronic diseases such as, chrohn's disease, epilepsy, anemia, dementia, spina bifida [7]. There are generally two different approaches to combine MIPs with a signal transduction unit (transducer): one is to immobilize a readymade MIP on a transducer using physical entrapment or chemical coupling [8], and the other to assemble an MIP layer in-situ on transducer surface [9]. The in situ assembly approach can be carried out on a small scale, and is often preferred when expensive chemicals (templates) are required. However, in most of the published procedures, the in situ polymerization has been reportedly found to be difficult to control and consequently, MIP film grafted on the transducer surface has large batch-to-batch variations. Although thick films, as obtained using different coating methods, contain plenty of binding sites, it is often difficult for the underlying transducer to detect binding events that take place beyond certain distance. In this study, we investigate a possible route to prepare ultra-thin MIP [poly (1,3,5-trisacrylamide-2,4,6-triazine-ethylene glycol dimethacrylate)] films using surface initiated AGET-ATRP. Polymer films are directly formed on the gold-coated quartz crystal resonator, which facilitates an easy monitoring of the polymer growth.

\section{Experimental}

\section{Reagents}

3-(mercaptopropyl) trimethoxysilane (MTS), (3chloropropyl)-trimethoxysilane (TMS), cupric chloride, 2,2'bi-pyridyl, triethyl amine (TEA), cholroform, ethylene glycol dimethacrylate (EGDMA), and other solvents were purchased from Sigma Aldrich Chemie (Steinheim, Germany). The stock solution of FA (500 $\left.\mathrm{mg} \mathrm{L}^{-1}\right)$ was prepared in triple-distilled water.

\section{Apparatus}

Microgravimetric measurements were performed by using $5 \mathrm{MHz}$ (AT-cut quartz crystals in a teflon holder and a quartz crystal analyzer) model. The following equation (Sauerbrey's equation) [10] has been established for an ATcut shear mode QCM:

$$
\Delta F=-2 F_{0}^{2}\left(\rho_{q} \mu_{q}\right)^{-1 / 2} \frac{\Delta m}{A}
$$

where $\Delta \mathrm{F}$ is the measured frequency shift due to the added mass in hertz, $F_{0}$ is the fundamental oscillation frequency of the dry crystal, $\Delta \mathrm{m}$ is the surface mass loading in grams, $\rho_{\mathrm{q}}$ is the density of quartz $\left(2.65 \mathrm{~g} \mathrm{~cm}^{-3}\right), \mu_{\mathrm{q}}$ is the shear modulus $\left(2.95 \times 1011 \mathrm{dyn}^{-2}\right)$, and $\mathrm{A}$ is the electrode area $\left(1.37 \mathrm{~cm}^{2}\right)$. For the $5 \mathrm{MHz}$ quartz crystals used in this work, Eq. (1) predicted that a frequency change of $1 \mathrm{~Hz}$ corresponds to a mass increase of $24.5 \mathrm{ng}$ on the electrode.
Morphological images were recorded using scanning electron microscope (SEM, JEOL, JSM, Model 840A, Netherlannds).

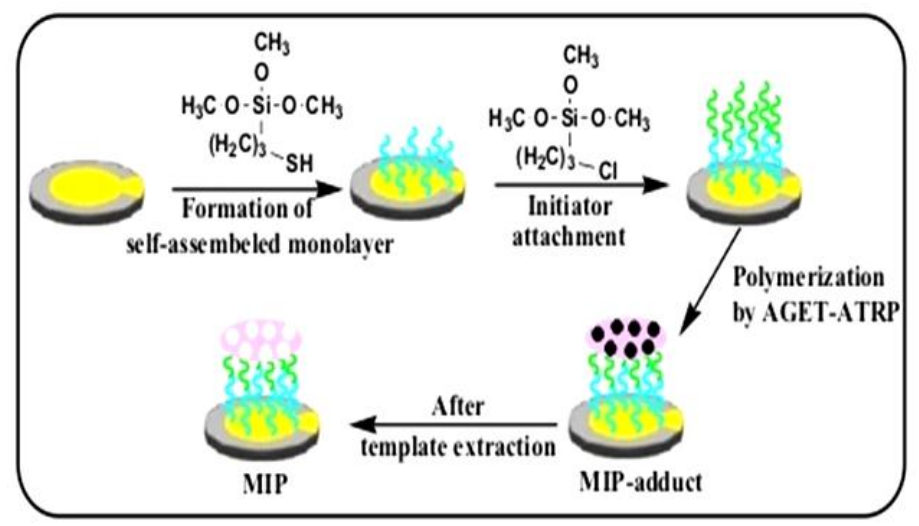

Fig. 1. Fabrication of MIP-modified QCM sensor using a surface grafted initiator.

\section{Preparation of the FA- imprinted QCM sensors}

Crystal was cleaned in a piranha solution (1:3, 30\% $\mathrm{H}_{2} \mathrm{O}_{2}$ /concentrated $\mathrm{H}_{2} \mathrm{SO}_{4}$ ) for $2 \mathrm{~min}$ before coating. The cleaned crystal was immersed into MTS $(0.30 \mathrm{mM}$ in ethanol) for $4 \mathrm{~h}$, in order to introduce thiol groups onto the gold surface of quartz crystal. The electrode was then washed with ethanol and deionized water for $10 \mathrm{~min}$ to remove the excess of thiols. Thus, a stable self-assembled monolayer of thiol was formed on the gold surface (Fig. 1). For the attachment of initiator to the SAM-modified gold surface, the SAM-modified crystal was again dipped in TMS $(0.5 \mathrm{mmol}$ in ethanol) for $6 \mathrm{~h}$ and then washed with ethanol and deionized water. For the polymerization, bpy $(0.02 \mathrm{mmol})$ and $\mathrm{CuCl}_{2}(0.02 \mathrm{mmol})$ were dissolved in 2 $\mathrm{mL}$ DMSO to obtain a solution of $\mathrm{Cu}$ (II)-complex. Subsequently, this complex was mixed with monomer (1,3,5 trisacrylamide 2,4,6 triazine, TAT, $0.2 \mathrm{mmol})$ [7], template (FA, $0.1 \mathrm{mmol})$ and EGDMA (4 mmol), in the presence of a reducing agent (TEA, $2 \mathrm{mmol}, 280 \mu \mathrm{L}$ ). Then, the reaction mixture was degassed and squeezed on initiator modified QCM sensor and finally the crystal was cured for $6 \mathrm{~h}$ at $65{ }^{\circ} \mathrm{C}$. The crystal was finally washed with $0.6 \mathrm{~mL}$ mixture of acetonitrile-triethylamine $(1: 4, \mathrm{v} / \mathrm{v})$, for the template extraction from MIP-FA adduct coating. (The QCM sensor coated with non-imprinted polymer [NIP, poly (1,3,5-trisacrylamide-2,4,6-triazine-ethylene glycol dimethacrylate)], having same polymer motif as that of MIP, was also prepared in the similar manner but, without template).

\section{Evaluation of sensor response}

The FA imprinted crystal was firstly stabilized in $600 \mu \mathrm{L}$ triple-distilled water at room temperature and a steady resonant frequency $\left(\mathrm{F}_{0}\right)$ was obtained. Then, the standard solution of FA in water $(600 \mu \mathrm{L})$ was dropped on the QCM chips and interacted for $20 \mathrm{~min}$. The frequency of the sensor was monitored until it became stable $\left(\mathrm{F}_{1}\right)$. The frequency shift for each concentration of FA was calculated using the equation: $\Delta \mathrm{F}=\mathrm{F}_{0}-\mathrm{F}_{1}$ and the evaluation was performed in triplicate. After each assay, FA was removed 
from the coating by extracting with a mixture of acetonitrile-triethylamine $(1: 4, \mathrm{v} / \mathrm{v})$. This washing process was repeated until the frequency of the sensor recovered to the $\mathrm{F}_{0}$ value.

\section{Results and discussion}

\section{Spectral characterization}

1H-NMR spectra of the monomer (TAT), template (FA), MIP-adduct and MIP were compared to study the binding interactions between the template and MIP. The peak due to MIP (amide protons) $6.8 \mathrm{ppm}$ shifted to $7.5 \mathrm{ppm}$, and the peaks due to template (-COOH, 9.5; - $\mathrm{OH} 10.8$ and 5.2 $\mathrm{ppm}$ ) were shifted to $10.0,11.2$, and $5.8 \mathrm{ppm}$, respectively after FA rebinding with MIP. Interestingly, these shifted peaks resumed their original positions after template extraction.

\section{Morphological characterization}

Fig. 2 shows the SEM images of MIP- and NIP- modified quartz crystal surfaces. It is clearly visible from $\mathbf{F i g} . \mathbf{2 b}$ that NIP surface is very smooth and compact in comparison to the MIP surface (Fig. 2a). The MIP surface is very porous (due to presence of several cavities), which afforded an uninterrupted access to analyte rebinding.
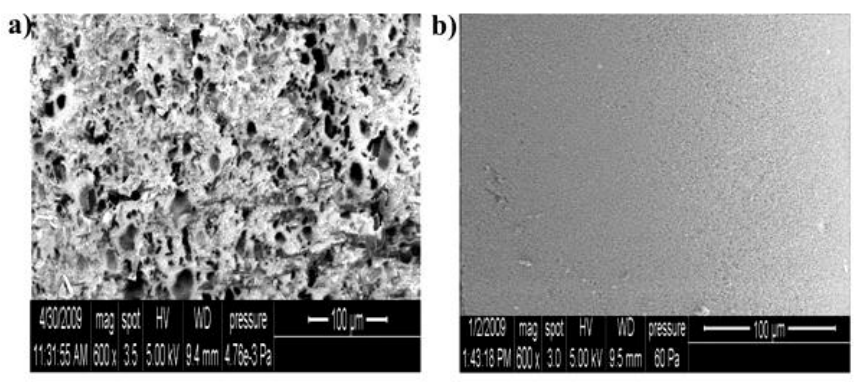

Fig. 2. SEM images of MIP (a) and NIP (b) modified QCM sensor.

\section{Sensor response}

The oscillator circuit in the instrumentation induced the quartz crystal to oscillate at its characteristic resonant frequency of $5 \mathrm{MHz}$. When the crystal was coated with the MIP, its oscillation frequency decreased, indicating an increase in the mass on the surface of the crystal. When the MIP-coated crystal was placed in a solution of FA, there was a further decrease in the oscillation frequency of the crystal. This decrease in frequency suggests an intake of FA by the polymer coating. This change in the oscillation frequency took place slowly, requiring about $20 \mathrm{~min}$ to reach a steady-state value. This behavior can be attributed to the characteristics of the kinetics involved in the diffusion and subsequent binding of the FA molecules in the cavities of the MIP coating. The frequency shift was reproducible and exhibited a relative standard deviation of $1.7 \%$ for $\mathrm{n}=3$ replicates. When the reference (i.e., NIPcoated) crystal was immersed in a solution of FA, a decrease in oscillation, very smaller than that exhibited by the MIP-coated crystal, was observed (Fig. 3a). This lowering of oscillation frequency could be attributed to some changes in the polymer coating (e.g., swelling). The frequency shift observed with the MIP-coated crystal was very sensitive to the concentration of the template solution. Fig. 3a, shows the calibration curve that relates the sensor response with the concentration of FA on MIP-modified crystal. Very good linearity $(r=0.999)$ was shown in the concentration range of 0.6 to $26.0 \mu \mathrm{g} \mathrm{L}^{-1}[\Delta \mathrm{F}(\mathrm{Hz})=$ $(1.826 \pm 1.129)+(40.527 \pm 0.330) \Delta \mathrm{m}(\mu \mathrm{g})]$ (Fig. 3b). The detection limit, defined as the concentration of analyte giving frequency shift equivalent to three standard deviation of the blank plus the net blank frequency shift, was $0.08 \mu \mathrm{g} \mathrm{L}^{-1}$. The NIP-coated sensor exhibited an erratic behavior with the different standard solutions of FA.

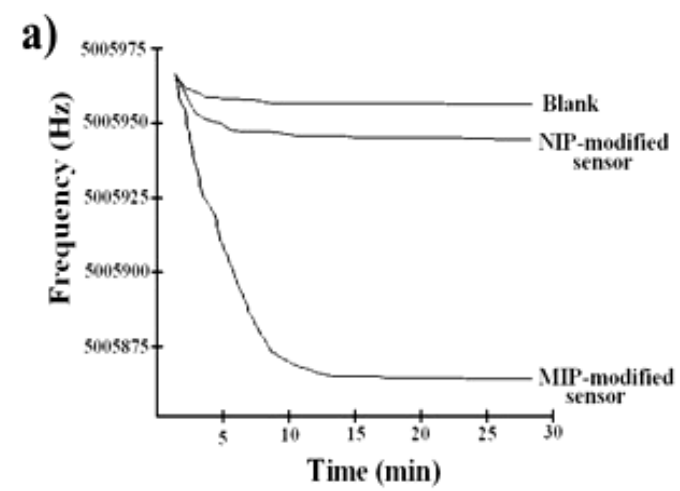

b)

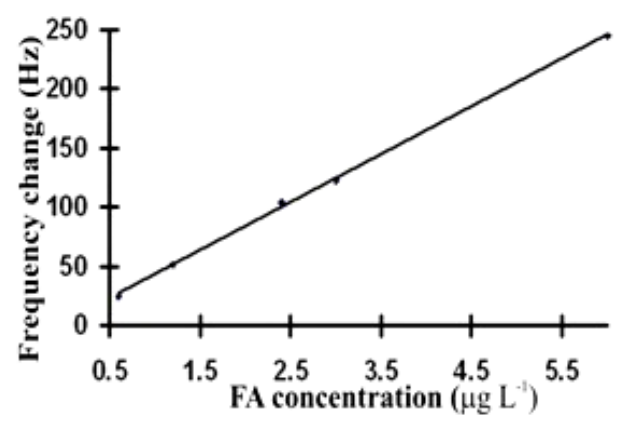

Fig. 3. (a) Response of the MIP and NIP-modified QCM sensor and (b) Calibration curve of FA concentration vs. frequency shift.

\section{Reusability and stability of sensor}

The regeneration of the coated QCM is of critical importance for application as a sensor. Reproducibility of the same MIP-QCM sensor was evaluated by measuring the response signal seven times in continuous manner which exhibited a better reproducibility, and the response signal of the sensor was about $1.6 \%$ loss at the 7 th cycle. In addition, our experiments indicated that the MIP-QCM sensor was quite stable which has proven its stability for more than three months with a slight loss of sensitivity $(1.1 \%)$. Any single sensor could be used for as many as 50 times with quantitative recoveries indicating reusability of the proposed sensor. Whereas, three sensors prepared in three different batches showed similar response corroborating the method reproducibility of sensor QCM modification. Interestingly, the NIP-modified QCM sensors also entailed similar magnitude of fabrication 
reproducibility and measurement precision as that shown by MIP-QCM sensors. This reflects the significance of molecular imprinting technique as demonstrated in this work. It can thus be concluded that the MIP-QCM sensor can be used many times without significant decrease of response signal.

\section{Conclusion}

By using living radical polymerization for the synthesis of a FA-imprinted film as antibody-mimic recognition material, a novel QCM sensor with good reproducibility, short response time, wide linear range, low detection limit, and high selectivity has been developed for the determination of FA at ultratrace level.

\section{Acknowledgement}

Authors thank Council of Scientific and Industrial Research (CSIR), New Delhi for the award of a senior research fellowship (to R.M.) and University Grant Commission (UGC) for research fellowships (to D. K. and M. P. T.).

\section{Reference}

1. Yang, Z.-P.; Zhang, C.-J. Sens. Actuators B 2009,142, 210. DOI: $10.1016 / j . s n b .2009 .08 .029$

2. Plomer, M.; Guilbault, G.G.; Hock, B. Enzyme and Microbial Technology 1992, 14, 230.

DOI: $10.1016 / 0141-0229(92) 90071-U$

3. Fung, Y.S.; Wong, Y.Y. Anal. Chem. 2001, 73, 5302. DOI: $10.1021 / a c 010655 y$

4. Marx, K.A. Biomacromolecules 2003, 4, 1099. DOI: $10.1021 / \mathrm{bm} 020116 \mathrm{i}$

5. Liu, F.; Liu, X.; Ng, S.C.; Chan, H.S. Sens. Actuators B 2006,113, 234 DOI: $10.1016 / j . s n b .2005 .02 .058$

6. Lin, T.Y.; Hu, C.H.; Chou, T.C. Biosens. Bioelectron. 2004, 20, 75. DOI: $10.1016 / \mathrm{j}$. bios.2004.01.028

7. Prasad, B. B.; Madhuri, R.; Tiwari, M. P.; Sharma, P. S. Sens. Actuators B 2010, 146, 321. DOI: $10.1016 / j . s n b .2010 .02 .025$

8. Ebarvia, B.S.; Binag, C.A.; Sevilla III, F. Anal. Bioanal. Chem. 2004, $378,1331$.

DOI: $10.1007 / \mathrm{s} 00216-003-2433-9$

9. Kugimiya, A.; Takeuchi, T. Biosens. Bioelectron. 2001, 16, 1059. DOI: $10.1016 / \mathrm{S} 0956-5663(01) 00227-5$

10. Sauerbrey, G. Z. Phys. 1959,155, 206 DOI: $10.1007 / \mathrm{BF} 01337937$

\section{AdVANCEd Materials Letters}

\section{Publish your article in this journal}

ADVANCED MATERIALS Letters is an international journal published quarterly. The journal is intended to provide top-quality peer-reviewed research papers in the fascinating field of materials science particularly in the area of structure, synthesis and processing, characterization, advanced-state properties, and applications of materials. All articles are indexed on various databases including $\underline{\mathrm{DOAJ}}$ and are available for download for free. The manuscript management system is completely electronic and has fast and fair peer-review process. The journal includes review articles, research articles, notes, letter to editor and short communications.

\section{Submit your manuscript: http://amlett.com/submitanarticle.php}

\section{JOURNAL}

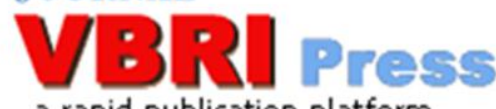

a rapid publication platform

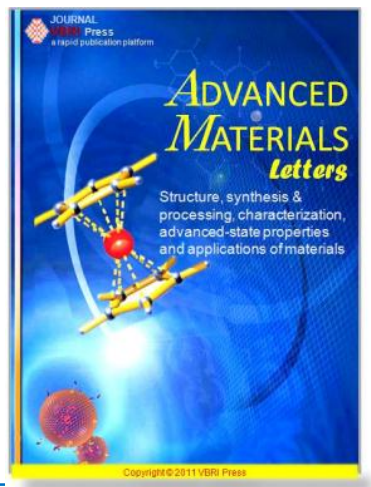

